\title{
RELIABILITY OF THE 50-FOOT WALK TEST AND 30-SEC CHAIR STAND TEST IN TOTAL KNEE ARTHROPLASTY
}

\author{
Bayram UnVer ${ }^{1}$, Serpil Kalkan ${ }^{1}$, ERtugrul Yuksel ${ }^{1}$, Turhan $K_{\text {ahraman }}{ }^{1}, V_{\text {asfi }} K_{\text {aratosun }}{ }^{1}$
}

\section{ABSTRACT}

Objective: To investigate the reliability of the 50-Foot Walk Test (50 FWT) and 30-second Chair Stand Test (30 CST) in patients who have undergone total knee arthroplasty (TKA). Methods: The study was designed as a test-retest research. Thirty-three patients who would undergo bilateral TKA were recruited. The tests 30 CST and 50 FWT were performed twice on the same day with 5-minute intervals, respectively. Between the first and second tests, patients waited for an hour on sitting position in order to prevent fatigue. In addition to these tests, we registered the knee pain experienced by the patients using a $100 \mathrm{~mm}$ VAS scale. Results: The 50 FWT and 30 CST showed excellent reliability.
ICC for 50 FWT and 30 CST were 0.97 and 0.92, respectively. SRD95 was 1.07 for 50 FWT and 0.96 for 30 CST. Conclusions: According to results of this study, both 50 FWT and 30 CST have excellent reliability in patients with TKA. These tests are simple, no time consuming and constitute sensitive methods to measure the functional performance in patients with TKA in the clinical settings. Clinicians and researchers may use these tests to quantify even small changes in functional performance for patients with TKA. Level of Evidence III, Diagnostic Study.

Keywords: Arthroplasty, Replacement, Knee. Performance tests, Rehabilitation.

Citation: Unver B, Kalkan S, Yuksel E, Kahraman T, Karatosun V. Reliability of the 50-foot walk test and 30-sec chair stand test in total knee arthroplasty. Acta Ortop Bras. [online]. 2015;23(4):184-7. Available from URL: http://www.scielo.br/aob.

\section{INTRODUCTION}

Objective assessment methods are essential for clinicians to determine the prognosis after total knee arthroplasty (TKA) at the decision-making process. Clinicians should use reliable and valid outcome measurement. ${ }^{1-4}$ In clinical studies, reliability is especially significant because stable data are required to make accurate statements about treatment effects or the amount of change observed over time. ${ }^{5}$

The most important parameters with TKA are improvement in pain and walking ability after treatment. $96.2 \%$ of patients underwent TKA expect improvement on walking ability. ${ }^{6}$ In addition, activities such as walking short distance and getting up from a chair are basic motor activities and are extremely important in terms of being independent for daily life. . $^{7-9}$ Thus assessment of daily living activities such as walking, getting up and down the stairs and sit up is significant in clinical practice. Daily living activities can be assessed either patient-reported or performance-based measure. Physical performance outcome measurements are complementary for functional evaluation of TKA patients. They involve the performance of specific activities associated with patient in order to evaluate performance abilities on a follow-up basis. ${ }^{10}$ However, current performance-based measurement systems (electromyography, force platforms, opto-electronic kinematic systems) are expensive, time-consuming and require sophisticated laboratories. . $^{8,11}$

Six-Minute Walk Test is widely used and well-defined measuring method to measure physical function in many diseases including TKA. ${ }^{12}$ The 6-Minute Walk Test is higly valuable test but longer walk tests (such as 6-Minute Walk Test) might overburden patients with OA. Thus, a shorter walk test (such as 50-Foot Walk Test (50 FWT)) is more clinically practical, and more appropriate..$^{13}$ Also, walking short distances (such as the 50 FWT) and sit-to-stand (such as the 30 CST) activities are recommended as the minimal core set of performance-based outcome measures in OA research and clinical practice. ${ }^{7}$ These tests are complementary to patient-report measures. They are recommended for use prospectively as outcome measures in research, and also in clinical practice to make treatment decisions based on the results and to monitor physical function of individual patients over time. ${ }^{7}$ Besides being simple methods for implementation and can be also implemented in a short time. These tests also require no special equipment, and can be performed in the examining room

All the authors declare that there is no potential conflict of interest referring to this article.

\section{Dokuz Eylul University, Balçova-Izmir, Turkey}

The study was conducted in Dokuz Eylul University Hospital, Balcova-izmir, Turkey.

Correspondence: Bayram UNVER, PhD, PT, Prof.Dokuz Eylul University, School of Physical Therapy and Rehabilitation, Department of Orthopedic Physiotherapy,

TR-35340, Balçova-Izmir-TURKEY. bayram.unver@deu.edu.tr 
as a part of every patient evaluation. In addition, these two tests are proper to assess daily living activities such as walking and sit up, frequently affected in patients with lower extremity disability, including in patients with TKA., ${ }^{2,7}$

The purpose of this study was to investigate the reliability of the 50 FWT and the 30 CST in patients who have undergone TKA.

\section{MATERIALS AND METHODS}

The study was designed as a test-retest research. Ethics approval for this study was obtained from the Ethics Committee of Dokuz Eylül University, Turkey. Informed consent was obtained from all participants in accordance with the Declaration of Helsinki.

For this study, 33 patients, who would underwent bilateral TKA, were recruited from July 2013 to December 2013. All the patients had been operated by the same surgeon using the paramedian approach. Patients were eligible if they were 18 years older above and have had a TKA at least 6 months ago. The exclusion criteria of the study were: revision TKA, unable to understand verbal and written instructions, experienced pain at rest of more than $50 \mathrm{~mm}$ on a visual analogue scale (VAS), having previously orthopaedic or neurological disorder that causes gait disturbance and having surgery within six months.

Demographic and clinical data of patients and the Hospital for Special Surgery (HSS) knee score was recorded. The HSS knee score criteria are based on a total of 100 points. The score consisted of seven categories: pain, function, Range of Motion, muscle strength, flexion deformity, instability and subtractions. Scores between 100 and 85 points are considered excellent results; scores between 84 and 70 points are good results; scores 69 and 60 points are fair, and scores less than 60 are considered poor results. ${ }^{14}$

50 FWT is a reliable measurement method and commonly used in studies of exercise based OA. ${ }^{7}$ Walking tests can be applied in two ways; normal self-selected walking speed and fast self-selected walking speed. However, fast self-selected walking speed method was shown more reliable in a previous study. ${ }^{15}$ Therefore; we preferred fast self-selected walking speed protocol in this study. In addition, walking 50 feet in one straight line was chosen to avoid the confounding influence of changing direction. ${ }^{15}$ All patients are asked to walk as quickly as possible in 50 feet length distance. Examiners counted number of steps and as soon as individual finish to walking 50 feet length distance, stopwatch was stopped and the time was recorded. ${ }^{2}$

30 CST is a reliable and valid measurement method for lower extremity strength assessment. ${ }^{16}$ According to the protocol published by Gill and McBurney, the chair with seat height of 17 inches was used. ${ }^{2}$ The same chair was used for all assessments. The starting position of subjects was standardized including buttock placement, back support, use of hands and foot placement. ${ }^{2}$ Arms were crossed at the wrists and held against the chest. The 30 CST patients were asked to sit and stand as quickly and safely as possible in 30 seconds. The total number of completed chair stands within 30 seconds is counted and recorded.

30 CST and 50 FWT were performed with 5-minute intervals, respectively. Patients performed twice trials for each tests on Acta Ortop Bras. 2015;23(4):184-7 the same day. Between the first trial and the second, patients waited for an hour on sitting position in order to prevent fatigue. During this period, patients were allowed to drink water, but not allowed to drink tea or coffee. Prior to each trial, we gave standardized verbal instructions to the patients on how to perform the test. Patients were allowed to use the assistive device (e.g. crutches, cane) to ensure safety but all patients did not use any assistive device.

In addition to these tests, we recorded knee pain level, using a 100-mm VAS. Before and after each test trial $100-\mathrm{mm}$ vertical VAS was used to assess knee pain.

\section{Statistical analysis}

All data were analyzed using Microsoft Excel Program (Microsoft Corp., Redmond, WA, USA) and the IBM ${ }^{\circledR}$ SPSS ${ }^{\circledR}$ Statistics (Version 15.0) software. The Kolmogorov-Smirnov/ Shapiro-Wilk tests were used for the determination of the normal distribution. Paired-samples $t$ test was used for comparing the difference between the first and second trials of the tests. The level of significance was determined $p<0.05$. The inter-class correlation coefficient (ICC) test was used for calculation of reliability between the two tests. The ICC $(2,1)$ model was used due to a single value that measures for each patient and the number of people selected from a large population of testers. ${ }^{12}$ The standard error of measurement (SEM) was calculated to ensure the accuracy of the measurement method. The smallest real difference at the $95 \%$ confidence level (SRD95) was calculated. ${ }^{10}$ SRD95 was obtained by multiplying the point estimate for the SEM by the $z=1.95$ for the 95\% confidence interval (i.e. SRD95 was calculated according to "SRD95 $=$ SEM $\times 1.95 \times \sqrt{2}$ " formula). A greater change than SRD95 was often interpreted as a true change.

\section{RESULTS}

Thirty three patients with bilateral TKA participated in the study. Characteristics of the patients are shown in the Table 1. The functional levels of the patients were perfect according to the HSS knee score $(87.60 \pm 9.17$ for right lower extremity and $87.69 \pm 9.23$ for left lower extremity).

In this study, 50 FWT and 30 CST showed excellent reliability. ICC for 50 FWT and 30 CST were 0.97 and 0.92 , respectively. SRD95 was 1.07 for 50 FWT. SRD95 was 0.96 for 30 CST. Reliability results of the tests were shown in the Table 2. There was no significant difference in mean VAS scores measured between the first trial and the second trial of 30 CST and 50 FWT $(p>0.05)$. No test trial was disqualified.

\section{DISCUSSION}

Outcome measurements must be valid, reliable, and responsive to changes in a patient's condition. Establishing the reliability of an outcome measure is a prerequisite to establishing meaningful data. It is important to examine the psychometric properties such as reliability of the 50 FWT and 30 CST in patients with TKA because reliability is population-specific. This is the first study that investigated reliability of 50 FWT and 30 CST in patients undergone TKA. According to results of this study, both 50 FWT and 30 CST have an excellent reliability in patients with TKA. 
Table 1. Characteristics of the patients.

\begin{tabular}{c|c}
\hline Variables & Mean SD or frequency (\%) n=33 \\
\hline Age (years old) & 66.969 .78 \\
\hline Sex - Female $\mathrm{n}(\%) /$ Male $\mathrm{n}(\%)$ & $27(\% 81.81) / 6(\% 18.19)$ \\
\hline Height (cm) & 158.217 .56 \\
\hline Weight (kg) & 81.1217 .20 \\
\hline Body Mass Index - kg/m² & 32.575 .62 \\
\hline $\begin{array}{c}\text { Hospital for Special Surgery } \\
\text { Knee Score- Right }\end{array}$ & 87.609 .17 \\
\hline $\begin{array}{c}\text { Hospital for Special Surgery } \\
\text { Knee Score- Left }\end{array}$ & 87.699 .23 \\
\hline
\end{tabular}

Table 2. The relative (ICC coefficient) and absolute (SEM and SRD95) reliability of the performance tests in patients with TKA.

\begin{tabular}{c|l|l|l|l|l|l}
\hline & $\begin{array}{c}\text { First Trial } \\
\text { [mean SD] }\end{array}$ & $\begin{array}{c}\text { Second Trial } \\
\text { [mean SD] }\end{array}$ & $\begin{array}{c}\text { Difference } \\
\text { [mean SD] }\end{array}$ & $\begin{array}{c}\text { ICC(2,1) } \\
(95 \% \text { Cl) }\end{array}$ & SEM & SRD95 \\
\hline $\begin{array}{c}30 \text { CST } \\
\text { (numbers) }\end{array}$ & 11.062 .34 & 11.572 .61 & $0.511 .27^{\star}$ & $\begin{array}{c}0.92 \\
(0.82 \text { to } 0.96)\end{array}$ & 0.35 & 0.81 \\
\hline $\begin{array}{c}50 \text { FWT } \\
\text { (seconds) }\end{array}$ & 25.398 .64 & 24.398 .16 & $1.002 .30^{*}$ & $\begin{array}{c}0.97 \\
(0.95 \text { to } 0.99)\end{array}$ & 0.39 & 0.91 \\
\hline
\end{tabular}

TKA: Total Knee Arthroplasty, 50 FWT: 50-Foot Timed Walk, 30 CST: 30-second Chair Stand Test, ICC intra-class correlation coefficient, Cl: confidence interval, SEM: standard error of measurement with a 95\% confidence interval, SRD95: smallest real difference at the $95 \%$ confidence level. ${ }^{*} p>0.05$

According to the HSS knee score, characteristics of functional level of the patients were excellent (HSS > 85). Thus, the homogeneity was provided in terms of the functional level of the patients.

Pain level in all patients has not been determined any significantly increase at the end of the tests according to VAS. This result suggests that clinicians can use these two tests safely without increasing pain levels and these tests can also be tolerated by patients.

Walking assessment after joint replacement provides crucial information about the healing process because walking is related to an active and independent lifestyle. ${ }^{12}$ Therefore, walking has to be assessed in TKA patients. However, longer walk tests (such as 6-minute walk test) require infrastructure and personnel, which are not always available in primary and population-based settings. Also, longer walk tests might overburden OA patients during the preoperative period, especially if they use walking aids, it may be more difficult to perform routinely in clinical practice. Therefore, a shorter walk test implemented in a short time is needed in clinical practice. The current study is the first study that investigated reliability of 50 FWT in patient undergone TKA and it was found that 50 FWT had ICC of 0.97 . Schilke et al. ${ }^{17}$ recruited 14 subjects with knee OA and when reassessed with the 50 FWT after one week. Schilke et al. ${ }^{17}$ found that 50 FWT had ICC of 0.97 for subjects with knee OA. In addition, Grace et al. ${ }^{15}$ showed that 50 FWT had ICC of 0.90 for 44 subjects with rheumatoid arthritis. Gill and McBurney ${ }^{2}$ showed that 50 FWT had an excellent reliability in people awaiting joint replacement surgery for the hip/knee osteoarthritis. Taking all these results into consideration, 50 FWT has an excellent reliability measurement method for subjects with rheumatoid arthritis, in people awaiting joint replacement surgery for the hip/knee osteoarthritis and also subjects with TKA.

Decreased ability to rise from a chair can limit independence or contribute to institutionalization.,16 The inability on rising up from sitting position is recognized by the World Health Organization as a disabling condition. Adequate quadriceps muscle strength is essential for the ability successfully to perform functional activities of arising from a chair. ${ }^{8}$ Moreover, Chair stand performance correlates with walking speed, independent ambulation and stair climbing. ${ }^{8,16}$ Chair stand test can be an indicator of lower extremity strength. Hughes et al. apud Schilke et al. ${ }^{18}$ showed that a person requires an average of $97 \%$ of lower extremity muscle strength for rising from a chair. In several studies, the CST was found to be adequate performance-based measure to assess functional changes after TKA implantation. ${ }^{8,18}$ In the current study 30 CST has an excellent reliability in patients with TKA. It was found that 30 CST had ICC of 0.92. Similarly, for the 30 CST, ICCs between 0.84 and 0.92 were found for community dwelling older adults. ${ }^{16}$ Gill and McBurney ${ }^{2}$ also reported ICCs between 0.95 and 0.98 for people awaiting joint replacement surgery for the hip/ knee osteoarthritis. Although there is contention about the magnitude of correlation required to ensure adequate reliability, clinically acceptable correlations have been suggested anywhere from 0.75 to $0.90 .^{2,19}$ Our results consistently satisfy the more stringent of these conditions with ICCs above 0.90 . The benefits of the simple chair stand test are that it requires no special equipment, it requires little additional time, and also it can be performed in the examining room as a part of every patient evaluation. ${ }^{8}$

To be useful in clinical practice, the obtained scores from outcome measures must be meaningful for clinicians. In this study, the SEM was used to identify the error associated with a patient's reported score and to estimate the value of SRD95 providing a threshold for interpreting the scores in the tests over time. ${ }^{20}$ These values are important while investigating the effect of interventions on functional performance in TKA. Because of our study's reliability is much higher and standard deviation is much smaller than these studies. Thus, 50 FWT and 30 CST for TKA patients is very sensitive and these two tests can detect the real differences on the patients' performances.

\section{CONCLUSION}

Reliability of 50 FWT and 30 CST are excellent for patients with TKA. These tests are inexpensive, simple, no time consuming and sensitive methods to measure the functional performance in patients with TKA in the clinical settings. Clinicians and researchers can use these two performance tests as complementary outcome measures for functional evaluation of TKA patients. Moreover, these tests can be used to quantify even small changes in functional performance after rehabilitation programs for patients with TKA. 


\section{REFERENCES}

1. Davies GM, Watson DJ, Bellamy N. Comparison of the responsiveness and relative effect size of the Western Ontario and McMaster Universities Osteoarthritis Index and the short-form medical outcomes study survey in a randomized, clinical trial of osteoarthritis patients. Arthritis Care Res. 1999;3:172-9.

2. Gill S, McBurney H. Reliability of performance-based measures in people awaiting joint replacement surgery of the hip or knee. Physiother Res Int. 2008;13(3):141-52.

3. Guyatt G, Walter S, Norman G. Measuring change over time: assessing the usefulness of evaluative instruments. J Chronic Dis 1987;2:171-8.

4. Husted JA, Cook RJ, Farewell VT, Gladman DD. Methods for assessing responsiveness: a critical review and recommendations. J Clin Epidemiol. 2000;53(5):459-68

5. Jones CJ, Rikli RE, Beam WC. A 30-s chair-stand test as a measure of lower body strength in community-residing older adults. Res $Q$ Exerc Sport. 1999;70(2):113-9.

6. Tekin B, Unver B, Karatosun V. Expectations in patients with total knee arthroplasty. Acta Orthop Traumatol Turc. 2012;46(3):174-80.

7. Dobson F, Hinman RS, Roos EM, Abbott JH, Stratford P, Davis AM, et al. OARSI recommended performance-based tests to assess physical function in people diagnosed with hip or knee osteoarthritis. Osteoarthritis Cartilage. 2013;21(8):1042-52.

8. Unver B, Karatosun V, Bakirhan S. Ability to rise independently from a chair during 6-month follow-up after unilateral and bilateral total knee replacement. J Rehabil Med. 2005;37(6):385-7.

9. Weiss JM, Noble PC, Conditt MA, Kohl HW, Roberts S, Cook KF, et al. What functional activities are important to patients with knee replacements? Clin Orthop Relat Res. 2002;(404):172-88.

10. Kennedy DM, Stratford PW, Wessel J, Gollish JD, Penney D. Assessing stability and change of four performance measures: a longitudinal study evaluating outcome following total hip and knee arthroplasty. BMC Musculoskelet Disord. 2005;28;6:3.
11. van den Dikkenberg N, Meijer OG, van der Slikke RM, van Lummel RC, van ieën $\mathrm{JH}$, Pijls B, et al. Measuring functional abilities of patientswith knee problems: rationale and construction of the DynaPort knee test. Knee Surg Sports Traumatol Arthrosc. 2002;10(4):204-12.

12. Unver B, Kahraman T, Kalkan S, Yuksel E, Karatosun V. Reliability of the six-minute walk test after total hip arthroplasty. Hip Int. 2013;23(6):541-5.

13. Unnanuntana A, Mait JE, Shaffer AD, Lane JM, Mancuso CA. Performance-based tests and self-reported questionnaires provide distinct information for the preoperative evaluation of total hip arthroplasty patients. J Arthroplasty. 2012;27(5):770-5.e1.

14. Narin S, Unver B, Bakırhan S, Bozan O, Karatosun V. Cross-cultural adaptation, reliability and validity of the Turkish version of the Hospital for Specia Surgery (HSS) Knee Score. Acta Orthop Traumatol Turc. 2014;48(3):241-8.

15. Grace EM, Gerecz EM, Kassam YB, Buchanan HM, Buchanan WW, Tugwell PS. 50-foot walking time: a critical assessment of an outcome measure in clinical therapeutic trials of antirheumatic drugs. $\mathrm{Br} \mathrm{J}$ Rheumatol. 1988;27(5):372-4.

16. Macfarlane DJ, Chou KL, Cheng YH, Chi I. Validity and normative data for thirty-second chair stand test in elderly community-dwelling Hong Kong Chinese. Am J Hum Biol. 2006;18(3):418-21.

17. Schilke JM, Johnson GO, Housh TJ, O'Dell JR. Effects of muscle-strength training on the functional status of patients with osteoarthritis of the knee joint. Nurs Res. 1996;45(2):68-72.

18. Mahoney OM, McClung CD, dela Rosa MA, Schmalzried TP. The effect of total knee arthroplasty design on extensor mechanism function. J Arthroplasty. 2002;17(4):416-21.

19. Hopkins WG. Measures of reliability in sports medicine and science. Sports Med. 2000;30(1):1-15

20. Almeida GJ, Schroeder CA, Gil AB, Fitzgerald GK, Piva SR. Interrater reliability and validity of the stair ascend/descend test in subjects with total knee arthroplasty. Arch Phys Med Rehabil. 2010;91(6):932-8. 